\title{
Safety and tolerability of nintedanib in patients with idiopathic pulmonary fibrosis in the USA
}

To the Editor:

Nintedanib has been approved for the treatment of idiopathic pulmonary fibrosis (IPF) in more than 60 countries, including the USA [1]. In the two phase III INPULSIS trials, nintedanib reduced disease progression by reducing decline in forced vital capacity [2]. Most patients were able to manage the side-effects of nintedanib, with $19.3 \%$ of patients treated with nintedanib versus $13.0 \%$ treated with placebo permanently discontinuing the study medication due to adverse events. The most frequent adverse events were gastrointestinal, particularly diarrhoea. The proportion of patients who had one or more serious adverse events was similar between nintedanib and placebo (30.4\% versus $30.0 \%)$.

Following the launch of nintedanib as a treatment for IPF, information on its safety and tolerability in the real-world setting has been collected via post-marketing surveillance. Here, we report an analysis of data collected in the USA between the launch of nintedanib on October 15, 2014, and October 31, 2015. Data on adverse events in patients with IPF treated with nintedanib, irrespective of causality, were collected via proactive communications with specialty pharmacies and a spontaneous reporting system. In addition, reports of adverse events were collected via direct contact with patients and caregivers as part of a patient support programme (OPEN DOORS). Adverse events were coded according to the Medical Dictionary for Regulatory Activities (MedDRA). Based on the mechanism of action of nintedanib and data from the INPULSIS trials, adverse events of interest in the post-marketing surveillance data were defined as diarrhoea, bleeding, hepatic disorders, arterial hypertension, major adverse cardiovascular events (MACE), myocardial infarction and stroke. For the purposes of this post-marketing safety analysis, the rates of these adverse events were assessed using the same definitions as used in the INPULSIS trials. Epidemiological data on the incidence of diarrhoea (in the general population) and other adverse events of interest (in unmatched patients with IPF) were obtained from the literature [3-9] and from a proprietary research database of patients representative of the commercially insured population of the USA (data on file, Optum Research Database).

Our post-marketing surveillance data came from 6758 patients with IPF treated with nintedanib. Median duration of exposure was 113 days (range 6-390 days). Estimated cumulative exposure was 2715 patient-years. Diarrhoea (2858 events, not individuals reporting at least one event), nausea (1476 events) and vomiting (705 events) were the most frequent adverse events. Most (95.0\%) adverse events were not serious. A total of 4062 adverse events defined as of interest in the post-marketing surveillance data were reported. Of these, 322 had a fatal outcome, including 27 cases of MACE, eight of myocardial infarction, three of stroke and two of bleeding. In 123 cases, the cause of death was not reported. The most frequent causes of death that were reported were progression of IPF (81 cases), respiratory failure (27 cases) and pulmonary fibrosis (18 cases).

The incidence of diarrhoea in the post-marketing surveillance data was 1053 per 1000 patient-years. Understanding that diarrhoea is a very common side-effect of nintedanib therapy, this was lower than the rate reported in INPULSIS (1331 per 1000 patient-years) and similar to the rate in epidemiological data from the general population (980 per 1000 patient-years) [5, 8] (figure 1a).

Based on its inhibition of the vascular endothelial growth factor receptor, nintedanib may increase the risk of bleeding. Patients at known risk of bleeding, including those treated with full-dose anticoagulants or

@ERSpublications

The safety and tolerability profile of nintedanib in patients with IPF in the clinical setting is consistent with the product label http://ow.ly/Nulb30jPAld

Cite this article as: Noth I, Oelberg D, Kaul M, et al. Safety and tolerability of nintedanib in patients with idiopathic pulmonary fibrosis in the USA. Eur Respir J 2018; 52: 1702106 [https://doi.org/10.1183/ 13993003.02106-2017]. 
a) Incidence rate per 1000 patient-years

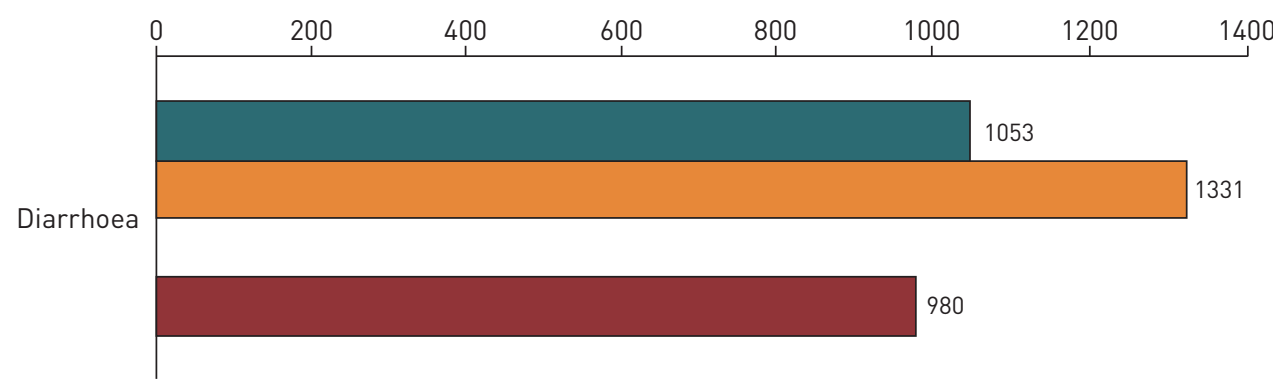

Post-marketing surveillance $\quad \square$ INPULSIS trials

Epidemiological data from general population

b)

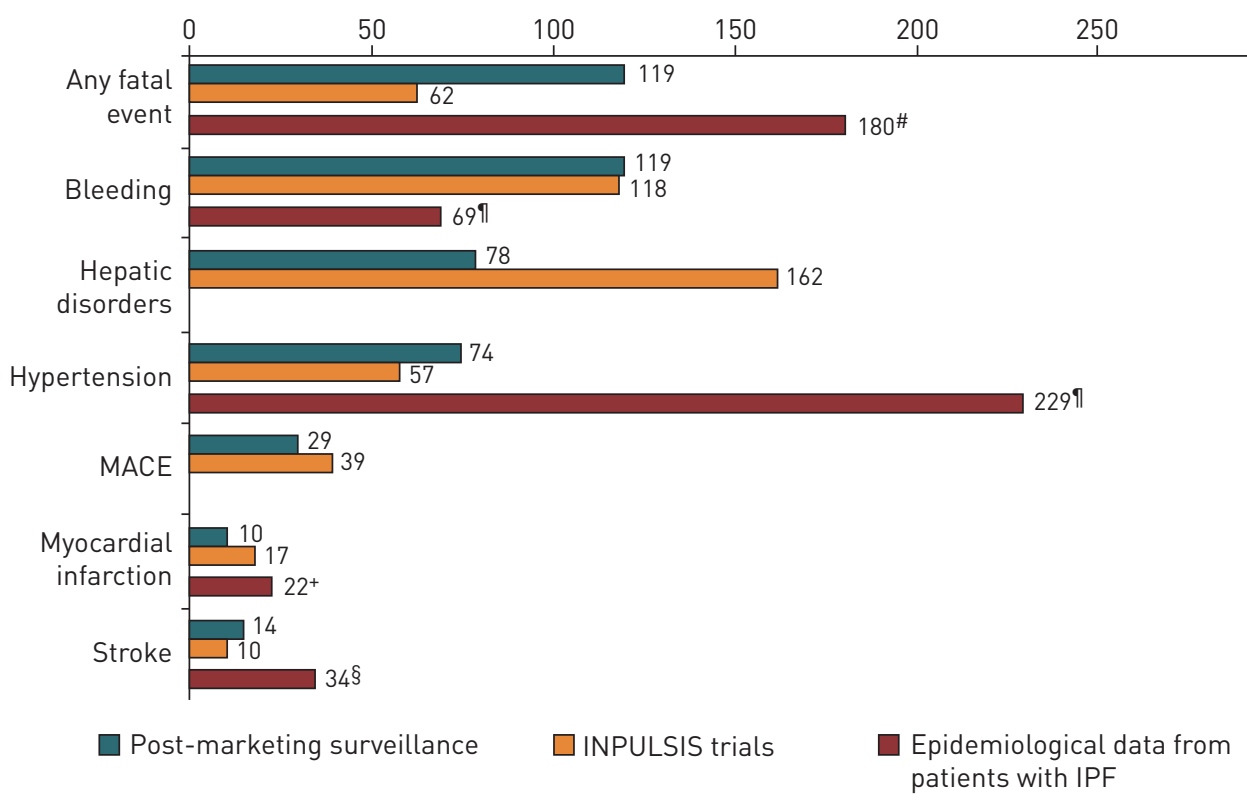

FIGURE 1 a) Incidence rates of diarrhoea across populations. b) Incidence rates of other adverse events of interest across populations. No data from epidemiological studies on hepatic disorders or major adverse cardiovascular events (MACE) were available from the published literature. \#: range 53-229 (from references $[3,6,7]$ and data on file, Optum Research Database); ${ }^{9}$ : data on file, Optum Research Database; ${ }^{+}$: range $14-$ 22 (from references $[4,7]$ and data on file, Optum Research Database); ${ }^{\S}$ : range 11-34 (from references $[7,9]$ and data on file, Optum Research Database).

high-dose antiplatelet therapy, were excluded from clinical trials, and the US product label specifies that patients at known risk of bleeding should receive nintedanib only if the anticipated benefit outweighs the potential risk [1]. The incidence of bleeding events in the post-marketing surveillance data was similar to that in INPULSIS (119 versus 118 per 1000 patient-years). Most bleeding events were not serious; the most frequent were epistaxis, contusion and rectal haemorrhage. The rate of bleeding events in patients with IPF based on healthcare claim data was lower than in the post-marketing surveillance data (figure 1b). This was not unexpected, as bleeding events that are not severe enough for a patient to seek medical attention (e.g. epistaxis) are generally not captured in claim-based data. Not surprisingly, known use of anticoagulants was higher in patients with bleeding than nonbleeding adverse events. Of 324 cases of bleeding in the post-marketing surveillance data, concomitant use of anticoagulants was reported in $37.7 \%$ and was unknown in $34.3 \%$ of cases. Of 4739 adverse events that were not bleeding, concomitant use of anticoagulants was reported in $25.4 \%$ and was unknown in $46.3 \%$ of cases.

In the INPULSIS trials, cardiac disorder adverse events were reported in $10.0 \%$ and $10.6 \%$ of patients treated with nintedanib and placebo, respectively, but there was a numerical imbalance in the proportion of patients with myocardial infarction based on the standardised MedDRA query "myocardial infarction" ( $2.7 \%$ versus $1.2 \%$, respectively) [10]. The US product label for nintedanib states that caution should be exercised in patients at higher cardiovascular risk [1]. The incidence of myocardial infarction in the post-marketing surveillance data (10 per 1000 patient-years) was lower than in INPULSIS (17 per 
1000 patient-years) or in epidemiological data from unmatched patients with IPF (22 per 1000 patient-years) (from references [4, 7] and data on file, Optum Research Database). The incidence of MACE was lower than in INPULSIS (29 versus 39 per 1000 patient-years) (figure 1b). The incidence of hypertension in the post-marketing surveillance data was similar to that in INPULSIS (74 and 57 per 1000 patient-years, respectively) and lower than in epidemiological data from unmatched patients with IPF (229 per 1000 patient-years) (data on file, Optum Research Database). The incidence of stroke reported in the post-marketing surveillance data was similar to that reported in INPULSIS (14 and 10 per 1000 patient-years, respectively) and lower than in epidemiological data from unmatched patients with IPF (34 per 1000 patient-years) (from references [7, 9] and data on file, Optum Research Database). The incidence of hepatic disorders (78 per 1000 patient-years) was lower than that in INPULSIS (162 per 1000 patient-years), perhaps reflecting less frequent measurement of liver enzymes.

Randomised controlled clinical trials provide the most robust data on the safety and tolerability of a drug in a specific patient population, but are necessarily of short duration and conducted in a population that is not fully representative of potential drug recipients. Post-marketing surveillance studies provide further data on the safety and tolerability of a drug, and help quantify rare adverse event rates [11-13]. However, post-marketing surveillance studies have a number of limitations, including under-reporting of non-serious adverse events $[12,14]$ and a lack of comprehensive documentation on adverse events, their outcomes (including dose adjustments and permanent discontinuations) and the characteristics of the patients who reported them. The relatively short median duration of exposure in this set of post-marketing data (113 days) should be noted as a limitation but data continue to be collected. Differences in methodologies mean that comparisons of data from post-marketing surveillance, clinical trials and epidemiological studies should be approached with caution. In addition, clinicians in practice may offer prescriptions with a safety bias, resulting in lower rates of certain adverse events in clinical practice than in clinical trials.

In conclusion, post-marketing surveillance data collected for $\sim 1$ year after the US launch of nintedanib as a treatment for IPF suggest that the safety and tolerability profile of nintedanib in the real-world clinical setting is consistent with the product label. As in clinical trials, the most frequent adverse events reported in clinical practice were non-serious gastrointestinal events, particularly diarrhoea.

Imre Noth $^{1}$, David Oelberg ${ }^{2}$, Manika Kaul ${ }^{3}$, Craig S. Conoscenti ${ }^{3}$ and Ganesh Raghu ${ }^{4}$

${ }^{1}$ University of Virginia School of Medicine, Charlottesville, VA, USA. ${ }^{2}$ Western Connecticut Health Network, Danbury Hospital, Danbury, CT, USA. ${ }^{3}$ Boehringer Ingelheim Pharmaceuticals, Inc., Ridgefield, CT, USA. ${ }^{4}$ Dept of Medicine, University of Washington, Seattle, WA, USA.

Correspondence: Imre Noth, University of Virginia, Dept of Medicine, Pulmonary and Critical Care, 1215 Lee Street, Charlottesville, VA 22908, USA. E-mail: IN2C@hscmail.mcc.virginia.edu

Received: July 032017 | Accepted after revision: April 202018

Acknowledgements: The authors would like to acknowledge the contribution of Alexandar Allinger, Boehringer Ingelheim Pharma GmbH \& Co. KG (Ingelheim am Rhein, Germany) to the acquisition and analysis of the data. Medical writing assistance was provided by Julie Fleming and Wendy Morris of FleishmanHillard Fishburn (London, UK), which was contracted and funded by Boehringer Ingelheim Pharmaceuticals, Inc. All authors were involved in the interpretation of the data and writing of this letter and have approved the final version.

Conflict of interest: M. Kaul is an employee of Boehringer Ingelheim. C.S. Conoscenti is an employee of Boehringer Ingelheim. G. Raghu reports receiving personal and other fees from Boerhinger Ingelheim, Sanofi and Vercyte, and other fees from BMS, Fibrogen, Gilead, Promedior, Roche-Genentech and UCB for acting as a consultant on IPF studies outside the submitted work. I. Noth reports receiving grants, and personal fees for study contracts, membership of a speaker bureau, membership of an advisory board and consulting from Boehringer Ingelheim; grants, and personal fees for study contracts, membership of a speaker bureau, membership of an advisory board and consulting from Genentech; personal fees for consulting from ImmuneWorks; and personal fees for membership of an advisory board from Sanofi outside the submitted work.

Support statement: The INPULSIS trials and the post-marketing surveillance programme for nintedanib were funded by Boehringer Ingelheim. Funding information for this article has been deposited with the Crossref Funder Registry.

\section{References}

1 Boehringer Ingelheim Pharmaceuticals, Inc. OFEVTM (nintedanib) prescribing information, 2017. http://bidocs. boehringer-ingelheim.com/BIWebAccess/ViewServlet.ser?docBase=renetnt\&folderPath=/Prescribing+Information/ PIs/Ofev/ofev.pdf Date last accessed: June 20, 2017.

2 Richeldi L, du Bois RM, Raghu G, et al. Efficacy and safety of nintedanib in idiopathic pulmonary fibrosis. $N$ Engl J Med 2014; 370: 2071-2082.

3 Gribbin J, Hubbard RB, Le Jeune I, et al. Incidence and mortality of idiopathic pulmonary fibrosis and sarcoidosis in the UK. Thorax 2006; 61: 980-985.

4 Hubbard RB, Smith C, Le Jeune I, et al. The association between idiopathic pulmonary fibrosis and vascular disease: a population-based study. Am J Respir Crit Care Med 2008; 178: 1257-1261. 
5 Lau JY, Sung J, Hill C, et al. Systematic review of the epidemiology of complicated peptic ulcer disease: incidence, recurrence, risk factors and mortality. Digestion 2011; 84: 102-113.

6 Navaratnam V, Fleming KM, West J, et al. The rising incidence of idiopathic pulmonary fibrosis in the UK. Thorax 2011; 66: 462-467.

7 Collard HR, Ward AJ, Lanes S, et al. Burden of illness in idiopathic pulmonary fibrosis. J Med Econ 2012; 15: 829-835.

8 Tam CC, Viviani L, Rodrigues LC, et al. The second study of infectious intestinal disease (IID2): increased rates of recurrent diarrhoea in individuals aged 65 years and above. BMC Public Health 2013; 13: 739.

9 Dalleywater W, Powell HA, Hubbard RB, et al. Risk factors for cardiovascular disease in people with idiopathic pulmonary fibrosis: a population-based study. Chest 2015; 147: 150-156.

10 Corte T, Bonella F, Crestani B, et al. Safety, tolerability and appropriate use of nintedanib in idiopathic pulmonary fibrosis. Respir Res 2015; 16: 116.

11 Suvarna V. Phase IV of drug development. Perspect Clin Res 2010; 1: 57-60.

12 Talbot JCC, Nilsson BS. Pharmacovigilance in the pharmaceutical industry. Br J Clin Pharmacol 1998; 45: 427-431.

13 Food and Drug Administration. Guidance for Industry. Good Pharmacovigilance Practices and Pharmacoepidemiologic Assessment, 2005. https://www.fda.gov/downloads/drugs/guidancecomplianceregulatoryinformation/guidances/ ucm071696.pdf Date last accessed: May 5, 2016.

14 Hazell L, Shakir SA. Under-reporting of adverse drug reactions: a systematic review. Drug Saf 2006; 29: 385-396.

Copyright (C)ES 2018 